\title{
Crystallization of Novel Polyglycine Hydrolases from two Fungal Families: Epicoccum sorghi and Fusarium solani
}

\author{
Nicole V. Fraser ${ }^{1}$, Marcia M. Chaudet ${ }^{1}$, Todd A. Naumann², Neil P.J. Price ${ }^{2}$, and David R. Rose ${ }^{1}$ \\ ${ }^{1}$ Department of Biology, University of Waterloo, Canada \\ ${ }^{2}$ ARS-NCAUR, USA
}

Polyglycine hydrolases (PGH) are secreted endoproteases that cleave glycine-glycine peptide bonds. Some PGH proteases specifically disrupt ChitA chitinase through recognition of the Nterminal chitin-binding domain. Subsequent cleavage occurs at the linker region between the chitin-binding domain and the chitinase domain. The cleavage activity is dependent on the presence of the SXXF serine motif. Two currently identified PGH proteases are ES-chitinase modifying protein (ES-Cmp) and FS-chitinase modifying protein (FS-Cmp), secreted by Epicoccum sorghi and Fusarium solani respectively. Despite minimal sequence and motif identity between ES-Cmp and FS-Cmp, both proteases share analogous cleavage activity deviating only in specific glycine recognition. Modelling software has been utilized to generate predictive structures for both proteases, based on solved structures in the PDB. Preliminary crystal conditions have been determined and reproduced utilizing the hanging drop vapour diffusion technique. These conditions provide the foundation for structural determination of the proteases. Current work is focused on co-crystallizing the proteases with a synthetic peptide substrate for determining structural differences and similarities between ES-Cmp and FS-Cmp during cleavage activity. 